\title{
Merging multisatellite precipitation products using stacking and EMOS-CSG methods
}

Tuantuan Zhang

Zhongmin Liang ( $\nabla$ zmliang@hhu.edu.cn )

Hohai University

Haijun Wang

Jun Wang

Yiming Hu

Binquan Li

\section{Research Article}

Keywords: Multisatellite precipitation merging, stacking, EMOS-CSG, gauge density

Posted Date: February 21st, 2022

DOI: https://doi.org/10.21203/rs.3.rs-1308502/v1

License: (9) This work is licensed under a Creative Commons Attribution 4.0 International License.

Read Full License 


\title{
Merging multisatellite precipitation products using stacking and
}

\section{EMOS-CSG methods}

\author{
Tuantuan Zhang ${ }^{1}$, Zhongmin Liang ${ }^{1}$, Haijun Wang ${ }^{2}$, Jun Wang ${ }^{1}$, Yiming $\mathrm{Hu}^{1}$, Binquan $\mathrm{Li}^{1}$ \\ ${ }^{1}$ College of Hydrology and Water Resources, Hohai University, Nanjing 210098, China \\ ${ }^{2}$ Shandong Hydrological Center, Jinan 250002, China \\ Corresponding author. Zhongmin Liang, Email: zmliang@ @hu.edu.cn
}

\section{Abstract:}

Precipitation is a fundamental element of hydrological analyses, water resource management, and drought monitoring. To obtain high-spatiotemporal-resolution precipitation information, in this study, we proposed a merging method to integrate four satellite precipitation products (i.e., the Tropical Rainfall Measuring Mission (TRMM) Multisatellite Precipitation 3B42 in Real-Time (TMPA-3B42RT), Climate Precipitation Center Morphing Technique (CMORPH), Global Satellite Mapping of Precipitation Near-Real-Time (GSMaP_NRT) and Precipitation Estimation from Remotely Sensed Information using Artificial Neural Networks (PERSIANN) products) with gauge observations. First, geographically weighted regression (GWR) was adopted to downscale the four original satellite products. Second, six base learners of stacking algorithm were used to correct the deviation in each downscaled satellite product. Third, the six corrected results of each downscaled product were integrated by a secondary learner of stacking algorithm. Fourth, the ensemble model output statistics-censored, shifted gamma (EMOS-CSG) method was adopted to produce the final precipitation product by merging the four satellite product-stacking results. The merging method was applied to the Beimiaoji basin from April to October in the 2016-2019 period. The results showed that (1) the daily merged precipitation product had a better performance than the original satellite products in terms of the six considered statistical indexes, with the lowest root mean square error (RMSE) at $4.33 \mathrm{~mm}$ and the highest correlation coefficient (CC) at 0.64 ; (2) the utilized merging method not only increased the spatial resolution to $1 \mathrm{~km}$ but also captured more detailed precipitation distribution information; and (3) considering the influence of the gauge density, the quality of the merged product was not further influenced after the number of gauge stations surpassed 16 .

Keywords: Multisatellite precipitation merging, stacking, EMOS-CSG, gauge density

\section{Introduction}

Precipitation plays an important role in hydrological models (Zhao et al. 2015), flood and drought monitoring (Breugem et al. 2020; Wang et al. 2019), and water resource management (Yang et al. 2017). However, due to the strong spatiotemporal variability in precipitation and climate change (Huang et al. 2016; Xie et al. 2019), accurately obtaining high-quality precipitation information remains a serious challenge. Precipitation data can be obtained in four ways, from gauges, weather radar, satellites, and reanalysis products; each of these products has its strengths and weaknesses. Gauge precipitation information can provide accurate long-sequence data, but the sparse density of gauges cannot capture the spatiotemporal distribution of precipitation (Verdin et al. 2015; Yin et al. 2021). Weather radar can capture precipitation information at a high spatiotemporal resolution, but the resulting precipitation information is subjected to large biases due to the influence of complex terrain and climate conditions (Wehbe et al. 2020). With the rapid development of remote sensing technology, satellite products can ensure near-global coverage, especially in gauge-sparse regions (Chao et al. 2018). Compared to weather radar, the public can freely obtain satellite products, such as the Tropical 

Estimation from Remotely Sensed Information using Artificial Neural Networks (PERSIANN), Integrated Multisatellite Retrievals for Global Precipitation Measurement (IMERG), and Climate Precipitation Center Morphing Technique (CMORPH) products. However, the accuracy of satellite-derived precipitation information is highly constrained by the utilized retrieval algorithm, cloud properties, and sensors (Chen et al. 2020). Reanalysis products (i.e., the European Centre for Medium-Range Weather Forecasts (ECMWF) Reanalysis v5 product (ERA5); Tarek et al. 2020) that combine ground and high-altitude observation data with historical atmospheric results through data-assimilation techniques can provide high-spatiotemporal-resolution historical datasets with continuous long-term series, but systematic error may constrain the performances of these datasets. To improve the accuracy of precipitation estimates and obtain realistic spatial distribution characteristics, merging precipitation datasets from different sources has become a common approach over the past few decades (Beck et al. 2017; Xu et al. 2020; Shao et al. 2021).

Traditional precipitation-merging methods include the Kalman filter (Todini 2001), the mean bias correction (Nerini et al. 2015), and kriging with external drift (Goudenhoofdt and Delobbe 2009); in these methods, gauge data are mainly merged with a single precipitation dataset. Although the performance of these techniques has been shown to be encouraging, fully capturing the spatiotemporal distribution of precipitation is still challenging. To address this problem, some advanced merging methods based on multisource precipitation have been developed in the last 10 years, such as the dynamic clustered Bayesian model averaging (DCBA) (Rahman et al. 2020), weighted average least square (WALS) (Rahman et al. 2020), and generalized three-cornered hat (TCH) methods (Xu et al. 2020). Compared to the use of a single dataset, multisource precipitation products provide more reliable precipitation distribution information and effectively improve the accuracy of the resulting merged products. Ma et al. (2018) introduced dynamic weights into Bayesian model averaging (BMA) and proposed the dynamic Bayesian model averaging approach to merge four precipitation products (i.e., TMPA-3B42RT, 3B42V7, CMORPH and PERSIANN-CDR) at a $0.25^{\circ}$ daily grid scale. The results showed that the merged product performed better than the other analyzed datasets. Yin et al. (2021) proposed a three-stage blending approach to combine three satellite products (i.e., IMERG Final, TMPA 3B42V7 and PERSIANN-CDR) with the ERA5 atmospheric reanalysis product; the conclusion was consistent with the previous case. Although the above studies effectively improved the quality of precipitation simulations, due to the direct merging of original datasets, merged coarse-resolution products are not sufficient for hydrological or meteorological applications (Chen et al. 2020).

In addition to the above merging methods, machine learning (ML) algorithms, which have the advantages of strong self-learning abilities and can deal with nonlinear problems, have been widely used in precipitation-merging applications. Common algorithms include the random forest (RF), support vector machine (SVM), artificial neural network (ANN), and extreme gradient boosting (XGB) algorithms. Zhang et al. (2021) developed a novel double machine learning (DML) method to merge four satellite products with gauge observations based on RF, ANN, SVM, and extreme learning machine (ELM) methods and then compared the performances of these different ML algorithms. Wu et al. (2020) employed a convolutional neural network (CNN) and long-short-term memory network (LSTM) to obtain high-spatiotemporal-resolution precipitation data in China by merging a TRMM 3B42 V7 satellite product with gauge data. Overall, it is feasible to filter out the best algorithm from a variety of ML algorithms or adopt a new algorithm for merging. However, the performance of ML 
superior to all others in every situation, and few efforts have been made to utilize the advantages of different ML algorithms when merging precipitation products.

In this study, we proposed a merging method to integrate four satellite precipitation products by adopting three algorithms, including geographically weighted regression (GWR), stacking, and the ensemble model output statistics-censored, shifted gamma (EMOS-CSG) algorithms. GWR was used to downscale the satellite products; stacking was used to integrate the advantages of different ML algorithms; and the EMOS-CSG algorithm was used to merge the integrated products. The proposed method was then adopted to evaluate the product-merging performance in the Beimiaoji Basin during the 2016-2019 period from April to October each year using six statistical indexes. In addition, we tried to assess the impact of the gauge density on the resulting merged products.

\section{Study area and data}

The Beimiaoji Basin is located in the upper reaches of the Huaihe River and has an area of 1711 $\mathrm{km}^{2}$. The topography of this basin transitions from high mountains in the south to hilly areas in the north. The basin is located in a transitional zone from a subtropical climate to a temperate monsoon climate, and the average annual rainfall total exceeds $1000 \mathrm{~mm}$; most precipitation is mainly concentrated in summer. Due to the influence of weather systems and topography, precipitation is unevenly distributed in space.

\section{Fig. 1 The distribution of 38 rain gauges in the study area; the green dots denote the training} gauge sites, and the red five-pointed stars show the test sites

A ground network of 38 gauges with available data from April to October in the 2016-2019 period was utilized in this study; this network provided daily precipitation observations and was considered to provide true values for this merging research. Among these stations, those denoted by green dots in Fig. 1 were used for training, and those indicated with red five-pointed stars were used for testing (Fig. 1).

Four satellite products covering quasiglobal precipitation were used in this study. The TMPA 3B42 in Real-Time (TMPA-3B42RT) product was merged with multichannel microwave and infrared (IR) data from all available satellites to produce optimal satellite precipitation estimates (Huffman et al. 2007). CMORPH was developed by using IR imagery to create motion vectors; then, the cloud motion vectors were applied to passive microwave (PMW) data to generate global precipitation information (Joyce et al., 2004; Deng et al., 2018). The Global Satellite Mapping of Precipitation (GSMaP) group was devoted to producing precipitation datasets with high precision (Kubota et al. 2007). The near-real-time product (GSMaP_NRT) produced by this group adopted a forward-only cloud advection technique and a Kalman filter to generate precipitation (Qi et al. 2021). PERSIANN was generated through the use of artificial neural networks (ANNs) to obtain rainfall rates from longwave IR brightness temperature images and parameters updated through PMW observations (Nguyen et al. 2019; Ashouri et al. 2015). Due to the inconsistent temporal resolutions among these four satellite products, the hourly precipitation information was averaged to the daily scale for the subsequent merging research. The spatiotemporal resolutions and data sources of the four satellite precipitation products can be found in Table 1 .

In addition to satellite products, topographic and meteorological variables closely related to precipitation were used in this study. Normalized difference vegetation index (NDVI) information was obtained from the MOD13A3 dataset of the National Aeronautics and Space Administration (NASA). Soil moisture came from the Soil Moisture Active-Passive (SMAP) dataset of NASA. The digital elevation model (DEM) dataset was downloaded from the Geospatial Data Cloud of China, and ArcGIS software was used to further extract topographic variables such as the latitude, longitude, elevation, 
slope, and aspect. Considering that there were no weather stations in the analyzed basin, meteorological variables such as temperature, atmospheric pressure, wind speed, and direction data were obtained from the ERA5-Land reanalysis dataset of the European Centre for Medium-Range Weather Forecasts (ECMWC). Soil moisture and meteorological variables with observation intervals shorter than 1 day were averaged to the daily scale. The above variables were resampled to the same spatial resolutions with the bilinear interpolation technique.

\section{Table 1 Spatiotemporal resolutions of remote sensing data and sources}

\section{Methods}

A flowchart elucidating the merging method used for the stacking process and the EMOS-CSG algorithm is presented in Fig. 2; this method was mainly divided into two processes: downscaling and merging. The GWR method developed by Brunsdon et al. (1996) can reflect the spatial nonstationary relationships between precipitation and its influencing factors and was used for downscaling in this study. Longitude, latitude, elevation, slope, and aspect, variables that are closely related to rainfall, were selected as explanatory variables. In addition, four original satellite precipitation datasets were separately downscaled to a $1-\mathrm{km}$ resolution; the specific operation steps can be found in Chen et al. (2020). The merging process was divided into three steps. In the first step, six base learners of stacking algorithm were adopted to correct the deviation in four satellite downscaled products (i.e., TMPA-3B42RT 1, CMORPH$_{1}$, GSMaP_NRT $_{1}$ and PERSIANN 1 ); each downscaled product produced six different correction results. In the second step, the corresponding six correction results were integrated by a secondary learner in the stacking algorithm for each product. In the third step, we merged the four integrated results (i.e., TMPA-3B42RT $2, \mathrm{CMORPH}_{2}, \mathrm{GSMaP}_{-} \mathrm{NRT}_{2}$ and PERSIANN ${ }_{2}$ ) using the EMOS-CSG algorithm and produced a final precipitation dataset at the daily, 1-km spatiotemporal resolution.

\section{Fig. 2 Framework elucidating the merging method used in this study}

\subsection{Stacking algorithm}

Stacking algorithm is a multimodel fusion technology that is commonly used in the field of machine learning; the algorithm is essentially a two-layer model (Breiman 1996; Sun and Trevor 2018). First, base learners, which mainly refer to different machine learning algorithms, are selected as the first layer in the model; the training datasets include gauge precipitation, downscaled satellite-derived precipitation, latitude, longitude, soil moisture, wind speed, wind direction, temperature, and atmospheric pressure information. Then, the output results of the first layer are regarded as the training data for the second layer of the model, and the final integration results are obtained. In terms of functionality, the second layer of the model can effectively combine the advantages of different base learners by assigning different weights to these learners to optimize the results. Taking the PERSIANN satellite product as an example, the structure of the stacking algorithm is illustrated in Fig. 2.

As the core of the stacking algorithm, base learners (machine learning algorithms) play an important role in precipitation merging, and each learner has its own advantages. RF algorithm, with has high computational efficiencies and low costs, can effectively solve the problem of overfitting (Breiman 2001). K-nearest neighborhood (KNN) algorithm is more effective in dealing with sample datasets with unbalanced spatial distributions (Hart 1968). XGB algorithm, which has strong generalization abilities and extensive portability characteristics, is commonly used in data science competitions (i.e., Kaggle) (Chen and Guestrin 2016). Light gradient boosting (LGB) algorithm can handle missing values with high-precision, fast-convergence, and parallel-computing performances (Ke et al. 2017). Categorical boosting (CatBoost) algorithm can overcome model overfitting and handle 
categorical features (Huang et al. 2019). Gradient-boosting machine (GBM) algorithm is based on decision trees and has the advantage of strong robustness (Friedman 2001).

Since latitude, longitude, elevation, slope, and aspect information are time-invariant, including these variables in machine learning training datasets results in data redundancy and decreases the merging skill. Therefore, meteorological variables (i.e., temperature, atmospheric pressure, and wind speed and direction) and soil moisture variables, which change over time, were selected to build the training dataset in this study. Considering the importance of spatial location information, latitude and longitude were also added to the training dataset.

\subsection{EMOS-CSG algorithm}

Gneiting et al. (2005) proposed the ensemble model output statistics (EMOS) method in 2005; this method is also known as nonhomogeneous regression. Based on the EMOS method, the distribution function of rainfall can be fitted by introducing a censored, shifted gamma (CSG) function to perform a postprocessing rainfall correction (Baran and Nemoda 2016; Javanshiri et al. 2021). In this study, we introduced this method into the precipitation-merging process. The probability density function (PDF) of the original gamma distribution can be expressed as follows:

$$
g_{k, \theta}(x)=\left\{\begin{array}{cc}
\frac{x^{k-1} \exp \left(-\frac{x}{\theta}\right)}{\theta^{k} \Gamma(k)} & x>0 \\
0 & x \leq 0
\end{array}\right.
$$

where $\Gamma(k)$ represents the value of the gamma function at $k$. The conversion relationships among the shape $(k)$, scale $(\theta)$, mean $(\mu)$ and variance $\left(\sigma^{2}\right)$ values of the gamma distribution can be expressed as follows:

$$
k=\frac{\mu^{2}}{\sigma^{2}}, \quad \theta=\frac{\sigma^{2}}{\mu}
$$

The mean and variance in the gamma distribution and in each satellite precipitation member $\left(f_{1} \ldots f_{N}\right)$ are connected as follows:

$$
\mu=a_{0}+a_{1} f_{1} \mathrm{~L}+a_{N} f_{N} \quad \sigma^{2}=b_{0}+b_{1} \bar{f}
$$

where $\bar{f}$ refers to the mean value of the satellite precipitation members; $a_{0} \ldots a_{N}$ denote the mean parameter values; and $b_{0}$ and $b_{1}$ represent the variance parameters, which can be calculated using the continuous-ranked probability score (CRPS) (Gneiting and Raftery 2007).

The cumulative distribution function $\left(\mathrm{G}_{\mathrm{k}, \theta, \delta}^{0}\right)$ of the CSG can be expressed as follows:

$$
\mathrm{G}_{\mathrm{k}, \theta, \delta}^{0}(x)=\left\{\begin{array}{cc}
\mathrm{G}_{\mathrm{k}, \theta}(x+\delta) & x \geq 0 \\
0 & x<0
\end{array}\right.
$$

where $\mathrm{G}_{\mathrm{k}, \theta}(x+\delta)$ is the cumulative distribution function of the original gamma distribution and $\delta$ indicates the offset to the left. The PDF $\left(g_{k, \theta, \delta}^{0}\right)$ can be estimated as follows:

$$
g_{k, \theta, \delta}^{0}(x)=\Pi_{\{x=0\}} G_{k, \theta}(\delta)+\Pi_{\{x>0\}}\left(1-G_{k, \theta}(\delta)\right) g_{k, \theta}(x+\delta)
$$

where $\Pi_{A}$ denotes an indicator function of $A$, and if $A \geq 1, \prod_{\{x>0\}}=1$; otherwise, $\prod_{\{x=0\}}=1$. Then, the mean $\lambda$ of CSG can be calculated as follows:

$$
\lambda=\theta k\left[1-G_{k, \theta}(\delta)\right]\left[1-G_{k+1, \theta}(\delta)\right]-\delta\left[1-G_{k, \theta}(\delta)\right]^{2}
$$

The quantile $\left(q_{p}\right)$ of $p$ in formula (4) is equal to 0 if $p \leq G_{k, \theta}(\delta)$; otherwise, $q_{p}$ can be 
calculated by $G_{k, \theta}\left(q_{p}+\delta\right)=p$.

The three gamma distribution parameters were the key to solving the EMOS-CSG algorithm; $k$ respectively; $\bar{P}$ and $\bar{O}$ denote the average satellite- and gauge-derived precipitation totals, respectively; $n$ is the number of samples; $N_{11}$ represents the number of times that both the gauge and satellite detected rainfall; $N_{10}$ denotes the number of times that only the satellite rather than the gauge detected rainfall; and $N_{01}$ is the number of times that only the gauge rather than the satellite detected rainfall. If the RMSE, MAE, and FAR values are close to 0 and the CC, FBI, and FH values are close to 1 , the merging effect is better. It is informative to note that a scatter plot should be used to determine whether the dot pitch is distributed around the $45^{\circ}$ line when the $\mathrm{CC}$ is adopted as a statistical index in order to discover whether the considered satellites provide systematically and $\theta$ could be calculated using formulas (2) and (3), and $\delta$ could be estimated using the CRPS. using formula (6). Considering that EMOS-CSG was calculated in grid units, the sparse rain-gauge network was interpolated to $1-\mathrm{km}$ grids using ordinary kriging; then, we obtained daily merged precipitation datasets at a $1-\mathrm{km}$ spatial resolution by merging the stacked results (i.e., TMPA-3B $42 \mathrm{RT}_{2}$, $\mathrm{CMORPH}_{2}, \mathrm{GSMaP}_{-} \mathrm{NRT}_{2}$, and PERSIANN ${ }_{2}$ ).

\subsection{Evaluation index}

To evaluate the performance of the merging results, six statistical indexes were selected, including the root mean square error (RMSE), correlation coefficient (CC), mean absolute error (MAE), false alarm ratio (FAR), frequency bias index (FBI), and frequency of hit $(\mathrm{FH})$; these indexes were calculated as follows:

$$
F H=\frac{N_{11}}{N_{11}+N_{10}}
$$

where $P_{i}$ and $O_{i}$ represent the satellite- and gauge-derived precipitation totals at the $i$ th point, overestimated or underestimated data. 


\section{Results and discussion}

\subsection{Performance of original and downscaled satellite precipitation products}

Due to the influence of the analyzed terrain, retrieval algorithms, and sensors, the accuracies of different satellite products may be different. Table 2 summarizes the evaluation statistics obtained for the original and downscaled satellite precipitation products. We found that the RMSE, MAE, FAR, and FH values of the four original satellite products did not differ extensively; the PERSIANN product had the lowest CC of 0.13 compared to the other three products, and the TMPA-3B42RT product had the lowest FBI value of 0.70 . Overall, the performances of the four satellite products revealed little differences within the analyzed basin, and no single product was significantly better than the other products; this finding was consistent with the research results reported by Mei et al. (2014) and Sun et al. (2018). Compared to the original products, the accuracies of the downscaled products were improved, but these improvements were not obvious; this result was related to the necessary residual-correction step in the downscaling process. The objective function of the residual-correction process was the satellite products instead of the gauges, leading to the limited accuracy improvements. Furthermore, the smaller CC (0.18-0.25) and larger RMSE (12.03-12.47 mm) values obtained indicated that substantial errors existed in the original and downscaled satellite precipitation products; thus, it was necessary to integrate gauge information to reduce these deviations.

\section{Table 2 Statistical metrics of original and downscaled satellite precipitation products}

\subsection{Performance assessments of merging products}

To test the effectiveness of the utilized merging method, we evaluated the accuracy of the analyzed satellite products at three different stages during the merging process; the evaluation statistics are listed in Table 3. First, the deviation-correction process involving base learners (machine learning algorithms) for the downscaled products significantly improved the satellite-derived precipitation accuracy. For instance, when ML algorithms were used, the MAEs decreased to 3.55-5.08 $\mathrm{mm}$ at a decreasing rate of 50-67\% compared to the downscaled products. The RMSEs decreased to 4.78-6.40 $\mathrm{mm}$ (by 47-62\%), and the CCs improved to 0.44-0.60. Compared to these substantial improvements in the RMSE, CC, and MAE, the improvement effects on the FAR, FBI, and FH indexes were not significant. All the above results demonstrated that ML algorithms could effectively reduce biases in satellite-derived precipitation products. Among the six ML algorithms, LGB performed the best overall, providing the highest $\mathrm{CC}$ of 0.60 and the lowest MAE of $3.55 \mathrm{~mm}$, followed by CatBoost, XGB, RF, KNN, and GBM. Furthermore, the three best-performing ML algorithms were all based on the improved gradient-boosting tree algorithm, indicating that these algorithms have certain advantages for merging precipitation in the analyzed basin.

After the first merging step, a secondary learner of stacking algorithm was employed to integrate the six ML results; this learner provided better scores than any of the ML algorithms discussed above. In addition, PERSIANN showed the best performance among the four satellite products, with the highest $\mathrm{CC}(\mathrm{FH})$ at $0.62(0.90)$ and the smallest MAE and RMSE values at 3.31 and $4.53 \mathrm{~mm}$, respectively. In terms of the correction effect, the stacking algorithm base learners greatly decreased the deviation in the results and avoided the uncertainty caused by using a single algorithm; additionally, the secondary learner integrated the advantages of the base learners and generated better correction results. Furthermore, the accuracy of the final merging product obtained by the third-stage EMOS-CSG method was further improved compared to the stacking results. The results described above demonstrated that the multisatellite precipitation merging method based on stacking and EMOS-CSG was effective and feasible. 
As they are the core of the precipitation-merging method, the utilized ML algorithms must effectively decrease the deviations in satellite-derived precipitation products. In this work, we also attempted to use other ML algorithms, including decision tree, support vector machine (SVM), logistic regression, extreme learning machine (ELM) and back-propagation (BP) network. Finally, the machine learning methods with the best merging effects among the methods listed above were selected as the base learners in the stacking algorithm. In terms of the algorithm structure, the stacking and EMOS-CSG processes integrated different datasets through weight-optimization processes. In the stacking process, weights were assigned to six machine learning results, while the EMOS-CSG method applied weights to the four satellite products (i.e., TMPA-3B42RT $2, \mathrm{CMORPH}_{2}, \mathrm{GSMaP}_{2} \mathrm{NRT}_{2}$ and PERSIANN $_{2}$ ). Fig. 3 displays the weights of the six machine learning algorithms applied to each product in the secondary learner step. The LGB algorithm accounted for the largest weights (over 23\%) for each product, followed by the CatBoost (20-22\%), XGB (16-20\%), RF (16-18\%), KNN (13-15\%) and GBM (3-4\%) algorithms. These results were consistent with the machine learning correction performance observed during the first merging stage (Table 3). In addition, to evaluate the sensitivities of topographic and meteorological variables to the base learner, we estimated the relative weight of each variable using an RF as an example. As shown in Fig. 4, all variables contributed valuable information. The satellite-derived precipitation variable accounted for the highest weight, indicating that this variable provided useful information for the precipitation-merging process. Temperature had the second-highest weight; this may have been related to the strong response of precipitation to temperature. The wind speed and direction may cause changes in atmospheric moisture, and the soil moisture signature can last a few hours to several days after a rain event (Maggioni and Massari 2018); thus, these variables showed similar performances in the RF algorithm. Although latitude and longitude provide important spatial location information, these variables have the characteristics of not changing with time, causing them to show the lowest weights in the precipitation-merging process.

Overall, the flexible merging framework described herein can not only select new ML algorithms (e.g., LSTM) as the base learners in the stacking process but can also add other precipitation datasets (e.g., reanalysis or weather radar datasets) to capture more rainfall information.

Table 3 Evaluation statistics of the precipitation products used for merging

Fig. 3 Weights of the six machine learning algorithms applied to each product in the secondary learner step

\section{Fig. 4 Weights of each variable in the training dataset applied to the RF algorithm}

\subsection{Assessment of the spatial distribution of precipitation}

Fig. 5 presents the spatial distributions of the downscaled satellite-derived products and the final merging product on June 20, 2017. Fig. 6 displays the corresponding scatter plots. According to the ribbon at the bottom of Fig. 5, the precipitation totals of TMPA-3B42RT, CMORPH, GSMaP_NRT, PERSIANN and the gauge network were mainly $16-40 \mathrm{~mm}, 32-64 \mathrm{~mm}, 48-80 \mathrm{~mm}, 10-45 \mathrm{~mm}$ and 100-220 mm, respectively. These values indicated that the four downscaled satellite precipitation products had obvious underestimations and large systematic errors. The same conclusion can be obtained from Fig. 6a-d, where the dot pitches of the corresponding scatter plots were below the $45^{\circ}$ line. After merging the four satellite products, the underestimation and mutation of the precipitation were significantly improved. The CC of the final merging product increased to 0.94 , and the dot pitch was evenly distributed around the $45^{\circ}$ line (Fig. 6e). In addition, the distribution of the merged precipitation product was consistent with that of the gauge observations, especially in the southern and 
central parts of the analyzed basin (Fig. 5e-f). This comparison further illustrated that the merging method had a good performance when estimating the spatial characteristics of precipitation.

Fig. 5 Spatial distributions of precipitation obtained from the (a) downscaled TMPA-3B42RT product, (b) downscaled CMORPH product, (c) downscaled GSMaP_NRT product, (d) downscaled PERSIANN product, (e) merged product, and (f) gauge network on June 20, 2017

Fig. 6 Scatter plots of the gauge- and satellite-derived precipitation measured for (a) the downscaled TMPA-3B42RT product, (b) downscaled CMORPH product, (c) downscaled GSMaP_NRT product, (d) downscaled PERSIANN product, and (e) merged products on June 20, 2017

\subsection{Sensitivity analysis of the gauge density}

The gauge density not only has an impact on the spatial pattern of the merged results but also affects the local deviation adjustment of satellite-derived precipitation products (Wang and Lin 2015; Bai et al. 2019; Nerini et al. 2015). In this study, the number of gauge stations in the training dataset was randomly reduced to $24,20,16,12,8$ and 4 while the test dataset remained unchanged. Then, we repeated the merging method with these different training dataset sizes and analyzed the gauge density sensitivity. Fig. 7 plots the change trends of the six analyzed statistical indexes under different gauge densities. As the gauge station density increased, the quality of the merged precipitation product improved. Taking a closer look, the increasing performance trend of the merged product began to significantly slow down when the number of gauges reached 16 stations. For example, when the gauge stations increased from 4 to 16, the RMSE (MAE, FAR) decreased by $2.25 \mathrm{~mm}$ (1.72 $\mathrm{mm}, 0.05)$; in contrast, the RMSE (MAE, FAR) decreased by only $0.33 \mathrm{~mm}(0.20 \mathrm{~mm}, 0.005)$ when the number of stations increased from 16 to 28 (Fig. 7a, 7c, 7e). These significant discrepancies indicated that the quality of the merged product was not influenced after the number of gauge stations in the Beimiaoji Basin surpassed 16.

Fig. 7 Merging accuracy statistics obtained under different rainfall station densities

\subsection{Discussion}

Despite the optimal performance of the merging method, some limitations in the process still require further study. First, we selected some topographic and meteorological variables as the explanatory variables in the GWR and ML algorithms according to previous studies; however, the rationality of these explanatory variables and the introduction of other new variables (i.e., cloud properties; Sharifi et al. 2019) require further analyses. For example, the introduction of the satellite-derived soil moisture variable may have generated increased uncertainty due to the systematic errors present in soil products. Second, the four satellite products considered in this study adopted space-borne sensors that use inversion algorithms to obtain precipitation information, while the reanalysis datasets (i.e., ERA5) were mainly obtained through data assimilation techniques. The use of two types of products generated based on different algorithms may result in more reliable precipitation spatial pattern estimates, so a reanalysis dataset could be added to the merging method to improve the quality of the merged product. Third, we only preliminarily studied the influence of the rainfall station density on the merging effect. Considering the small watershed area and relatively dense gauge stations in the study area, the gauge density threshold of 16 may not be suitable for other watersheds with sparse stations (i.e., those in Western China). In addition, due to the limited number of stations, only 28 stations were adopted to explore the gauge density sensitivity, and this value may have impacted the accuracy of the threshold results. Further efforts are thus needed to explore the gauge density threshold 
in different basins with more gauge stations.

\section{Conclusions}

In this study, we adopted GWR, stacking, and EMOS-CSG methods to merge multisatellite precipitation products and gauge observations. Four coarse-resolution satellite products (i.e., TMPA-3B42RT, CMORPH, GSMaP_NRT and PERSIANN) were adopted in combination with topographic and meteorological variables to produce a daily precipitation dataset with a 1-km resolution. The merging method was applied to the Beimiaoji basin from April to October each year during the 2016-2019 period. The RMSE, CC, MAE, FAR, FBI and FH were used to evaluate the performance of the merged products. Then, we tried to assess the impact of the gauge density on the merged products. The key findings of the study are as follows:

(1) The four original satellite precipitation products did not differ extensively in terms of the six statistical indexes. Although the spatial resolution of the products increased to $1 \mathrm{~km}$ through the use of the GWR method, the smaller CC (0.18-0.25) and larger RMSE (12.03-12.47) required gauge-derived and meteorological information to be integrated with the satellite products to reduce the deviations.

(2) The merging method based on stacking and EMOS-CSG significantly improved the performance of the precipitation products. The RMSE decreased to $4.33 \mathrm{~mm}$ and the CC improved to 0.64 compared to the average values obtained for the four original satellite precipitation products $(\mathrm{RMSE}=12.45 \mathrm{~mm}$ and $\mathrm{CC}=0.20)$.

(3) The stacking method could integrate the advantages of the base learners and greatly decrease the biases of the downscaled products. In addition, satellite precipitation and temperature accounted for relatively large weights according to the base learners.

(4) The performance of the merged product was affected by the gauge density. Increasing the gauge density could decrease the deviation in the results and improve the performance of the merged precipitation product, although the quality of the merged product was not further influenced after the number of gauge stations surpassed the threshold of 16.

Authors contribution Zhongmin Liang: conceptualization, methodology, funding acquisition; Tuantuan Zhang: data preparation, software, validation, visualization, writing - original draft; Haijun Wang: supervision; Jun Wang: supervision, data analysis; Yiming Hu: reviewing; Binquan Li: reviewing.

Funding This study was support by the National Natural Science Foundation of China (Grant No: 41730750) and National Natural Science Foundation of China (Grant No:41877147).

Availability of data and material Gauge precipitation data are collected by authors. The TMP A-3B42RT precipitation datasets are available at https://gpm.nasa.gov/data/directory. CMORPH d ata are available at https://rda.ucar.edu/datasets/ds502.1/index.html\#! access. GSMaP_NRT data are available at https://sharaku.eorc.jaxa.jp/GSMaP_CLM/index.htm. PERSIANN data are available at https://chrsdata.eng.uci.edu/. NDVI data are available at https://ladsweb.modaps.eosdis.nasa.gov /search/. Soil moisture data are available at https://nsidc.org/data/SPL4SMGP/versions/4. DEM d ata are available at http://www.gscloud.cn/. Wind speed, wind direction, atmospheric pressure an d temperature are available at https://cds.climate.copernicus.eu/cdsapp\#!/dataset/reanalysis-era5-lan $\mathrm{d} ? \mathrm{tab}=$ overview.

Code availability The code will be available upon request.

\section{Declarations}


Ethics approval This article is original and the manuscript has not been submitted to more than one journal for simultaneous consideration. The submitted work has not been published elsewhere before. The authors agreed on the publication ethics.

Consent to participate The authors have given their consent for submitting this paper to the Theoretical And Applied Climatology.

Consent for publication The authors agree to give permission to the publisher to publish their work.

Conflict of interest The authors declare no competing interests.

\section{References}

Ashouri H, Hsu K-L, Sorooshian S, Braithwaite DK, Knapp KR, Cecil LD, Nelson BR, Prat OP (2015) PERSIANN-CDR: Daily Precipitation Climate Data Record from Multisatellite Observations for Hydrological and Climate Studies. Bulletin of the American Meteorological Society 96(1):69-83.

Bai X, Wu X, Wang P (2019) Blending long-term satellite-based precipitation data with gauge observations for drought monitoring: Considering effects of different gauge densities. Journal of Hydrology 577: 124007.

Baran S, Nemoda D (2016) Censored and shifted gamma distribution based EMOS model for probabilistic quantitative precipitation forecasting. Environmetrics 27(5): 280-292.

Beck HE, Van Dijk AI, Levizzani V, Schellekens J, Miralles DG, Martens B, Roo AD (2017) MSWEP: 3-hourly $0.25^{\circ}$ global gridded precipitation (1979-2015) by merging gauge, satellite, and reanalysis data. Hydrology and Earth System Sciences 21(1): 589-615.

Breiman L (1996) Stacked regressions. Machine learning 24(1): 49-64.

Breiman L(2001) Random forests. Machine learning 45(1): 5-32.

Breugem A.J., Wesseling J.G., Oostindie K, Ritsema C.J. (2020) Meteorological aspects of heavy precipitation in relation to floods-an overview. Earth-Science Reviews 204:103171.

Brunsdon C, Fotheringham AS, Charlton ME (1996) Geographically weighted regression: a method for exploring spatial nonstationarity. Geographical analysis 28(4): 281-298.

Chao L, Zhang K, Li Z, Zhu Y, Wang J, Yu Z (2018) Geographically weighted regression based methods for merging satellite and gauge precipitation. Journal of Hydrology 558: 275-289.

Chen S, Xiong L, Ma Q, Kim JS, Chen J, Xu CY (2020) Improving daily spatial precipitation estimates by merging gauge observation with multiple satellite-based precipitation products based on the geographically weighted ridge regression method. Journal of Hydrology 589: 125156.

Chen T, Guestrin C (2016) Xgboost: A scalable tree boosting system. Proceedings of the 22nd acm sigkdd international conference on knowledge discovery and data mining 785-794.

Deng X, Nie S, Deng W, Cao W (2018) Statistical evaluation of the performance of gridded monthly precipitation products from reanalysis data, satellite estimates, and merged analyses over China. Theoretical and applied climatology 132(1): 621-637.

Friedman JH (2001) Greedy Function Approximation: A Gradient Boosting Machine. Annals of Statistics 29(5):1189-1232.

Gneiting T, Raftery AE, Westveld III AH, Goldman T (2005) Calibrated probabilistic forecasting using ensemble model output statistics and minimum CRPS estimation. Monthly Weather Review 133(5): 1098-1118.

Gneiting T, Raftery AE (2007) Strictly proper scoring rules, prediction, and estimation. Journal of the American Statistical Association 102(477): 359-378. 
Goudenhoofdt E, Delobbe L (2009) Evaluation of radar-gauge merging methods for quantitative precipitation estimates. Hydrology and Earth System Sciences 13(2): 195-203.

Hart P (1968) The condensed nearest neighbor rule. IEEE transactions on information theory 14(3): 515-516.

Huang G, Wu L, Ma X, Zhang W, Fan J, Yu X, Zeng W, Zhou H (2019) Evaluation of CatBoost method for prediction of reference evapotranspiration in humid regions. Journal of Hydrology 574: 1029-1041.

Huang S, Huang Q, Chen Y, Xing L, Leng G (2016) Spatial-temporal variation of precipitation concentration and structure in the Wei River Basin, China. Theoretical and applied climatology 125(1): 67-77.

Huffman GJ, Bolvin DT, Nelkin EJ, Wolff DB, Adler RF, Gu G, Hong Y, Bowman KP, Stocker EF (2007) The TRMM Multisatellite Precipitation Analysis (TMPA): Quasi-Global, Multiyear, Combined-Sensor Precipitation Estimates at Fine Scales. Journal of Hydrometeorology $8(1): 38-55$.

Javanshiri Z, Fathi M, Mohammadi SA (2021) Comparison of the BMA and EMOS statistical methods for probabilistic quantitative precipitation forecasting. Meteorological Applications 28(1): e1974.

Joyce RJ, Janowiak JE, Arkin PA, Xie P (2004) CMORPH: A Method that Produces Global Precipitation Estimates from Passive Microwave and Infrared Data at High Spatial and Temporal Resolution. Journal of Hydrometeorology 5(3):487-503.

Ke G, Meng Q, Finley T, Wang T. Chen W, Ma W, Ye Q, Liu T (2017) Lightgbm: A highly efficient gradient boosting decision tree. Advances in neural information processing systems 30: 3146-3154.

Kubota T, Shige S, Hashizume H, Aonashi K, Takahashi N, Seto S, Hirose M, Takayabu YN, Ushio T, Nakagawa K, Iwanami K, Kachi M, Okamoto K (2007) Global Precipitation Map Using Satellite-Borne Microwave Radiometers by the GSMaP Project: Production and Validation. IEEE Transactions on Geoscience and Remote Sensing 45(7):2259-2275.

Ma Y, Hong Y, Chen Y, Yang Y, Tang G, Yao Y, Long D, Li C, Han Z, Liu R (2018) Performance of optimally merged multisatellite precipitation products using the dynamic Bayesian model averaging scheme over the Tibetan Plateau. Journal of Geophysical Research: Atmospheres 123(2): 814-834.

Maggioni V, Massari C (2018) On the performance of satellite precipitation products in riverine flood modeling: A review. Journal of Hydrology 558: 214-224.

Mei Y, Anagnostou EN, Nikolopoulos EI, Borga M (2014) Error analysis of satellite precipitation products in mountainous basins. Journal of Hydrometeorology 15(5): 1778-1793.

Nerini D, Zulkafli Z, Wang LP, Onof C, Buytaert W, Lavado-Casimiro W, Guyot JL (2015) A comparative analysis of TRMM-rain gauge data merging techniques at the daily time scale for distributed rainfall-runoff modeling applications. Journal of Hydrometeorology 16(5): 2153-2168.

Nguyen P, Shearer EJ, Tran H, Ombadi M, Hayatbini N, Palacios T, Huynh P, Braithwaite D, Updegraff G, Hsu K, Kuligowski B, Logan WS, Sorooshian S (2019) The CHRS Data Portal, an easily accessible public repository for PERSIANN global satellite precipitation data. Sci Data 6:180296.

Qi W, Yong B, Gourley JJ (2021) Monitoring the super typhoon lekima by GPM-based near-real-time satellite precipitation estimates. Journal of Hydrology 603:126968

Rahman KU, Shang S, Shahid M, Wen Y, Khan AJ (2020) Development of a novel weighted average least squares-based ensemble multi-satellite precipitation dataset and its comprehensive evaluation 
over Pakistan. Atmospheric Research 246: 105133.

Rahman KU, Shang S, Shahid M, Wen Y, Khan Z (2020) Application of a dynamic clustered Bayesian Model Averaging (DCBA) algorithm for merging multisatellite precipitation products over Pakistan. Journal of Hydrometeorology 21(1): 17-37.

Shao Y, Fu A, Zhao J, Xu J, Wu J (2021) Improving quantitative precipitation estimates by radar-rain gauge merging and an integration algorithm in the Yishu River catchment, China. Theoretical and Applied Climatology 144(1): 611-623.

Sharifi E, Saghafian B, Steinacker R (2019) Downscaling satellite precipitation estimates with multiple linear regression, artificial neural networks, and spline interpolation techniques. Journal of Geophysical Research: Atmospheres 124(2): 789-805.

Sun Q, Miao C, Duan Q, Duan Q, Ashouri H, Sorooshian S, Hsu K (2018) A review of global precipitation data sets: Data sources, estimation, and intercomparisons. Reviews of Geophysics 56(1): 79-107.

Sun W, Trevor B (2018) A stacking ensemble learning framework for annual river ice breakup dates. Journal of Hydrology 561: 636-650.

Tarek M, Brissette FP, Arsenault R (2020) Evaluation of the ERA5 reanalysis as a potential reference dataset for hydrological modelling over North America. Hydrology and Earth System Sciences 24(5): 2527-2544.

Todini E (2001) A Bayesian technique for conditioning radar precipitation estimates to rain-gauge measurements. Hydrology and Earth System Sciences 5(2): 187-199.

Verdin A, Rajagopalan B, Kleiber W, Funk C (2015) A Bayesian kriging approach for blending satellite and ground precipitation observations. Water Resources Research 51(2): 908-921.

Wang XL, Lin A (2015) An algorithm for integrating satellite precipitation estimates with in situ precipitation data on a pentad time scale. Journal of Geophysical Research: Atmospheres 120(9): 3728-3744.

Wang Y, Li J, Zhang T, Wang B (2019) Changes in drought propagation under the regulation of reservoirs and water diversion. Theoretical and Applied Climatology 138(1): 701-711.

Wehbe Y, Temimi M, Adler RF (2020) Enhancing precipitation estimates through the fusion of weather radar, satellite retrievals, and surface parameters. Remote Sensing 12(8): 1342.

Wu H, Yang Q, Liu J, Wang G (2020) A spatiotemporal deep fusion model for merging satellite and gauge precipitation in China. Journal of Hydrology 584: 124664.

Xie W, Zeng X, Zhang S, Wu J, Wang D, Zhu X (2019) Bayesian evaluation of meteorological datasets for modeling snowmelt runoff in Tizinafu watershed in Western China. Theoretical and Applied Climatology 138(3): 1991-2006.

Xu L, Chen N, Moradkhani H, Zhang X, Hu C (2020) Improving global monthly and daily precipitation estimation by fusing gauge observations, remote sensing, and reanalysis data sets. Water Resources Research 56(3): e2019WR026444.

Yang N, Zhang K, Hong Y, Zhao Q, Huang Q, Xu Y, Xue X, Chen S (2017) Evaluation of the TRMM multisatellite precipitation analysis and its applicability in supporting reservoir operation and water resources management in Hanjiang basin, China. Journal of Hydrology 549: 313-325.

Yin J, Guo S, Gu L, Zeng Z, Liu D, Chen J, Shen Y, Xu C (2021) Blending multi-satellite, atmospheric reanalysis and gauge precipitation products to facilitate hydrological modelling. Journal of Hydrology 593: 125878.

Zhang L, Li X, Zheng D, Zhang K, Ma Q, Zhao Y, Ge Y (2021) Merging multiple satellite-based 
precipitation products and gauge observations using a novel double machine learning approach. Journal of Hydrology 594: 125969.

Zhao H, Yang S, Wang Z, Zhou X, Luo Y, Wu L (2015) Evaluating the suitability of TRMM satellite rainfall data for hydrological simulation using a distributed hydrological model in the Weihe River catchment in China. Journal of Geographical Sciences 25(2): 177-195. 


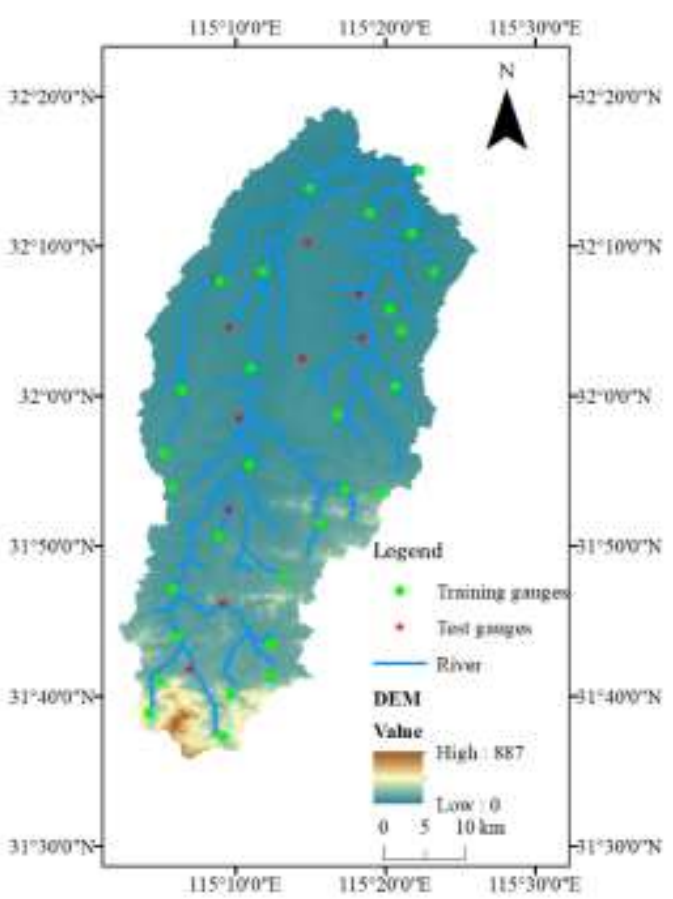

Fig. 1 The distribution of 38 rain gauges in the study area; the green dots denote the training gauge sites, and the red five-pointed stars show the test sites

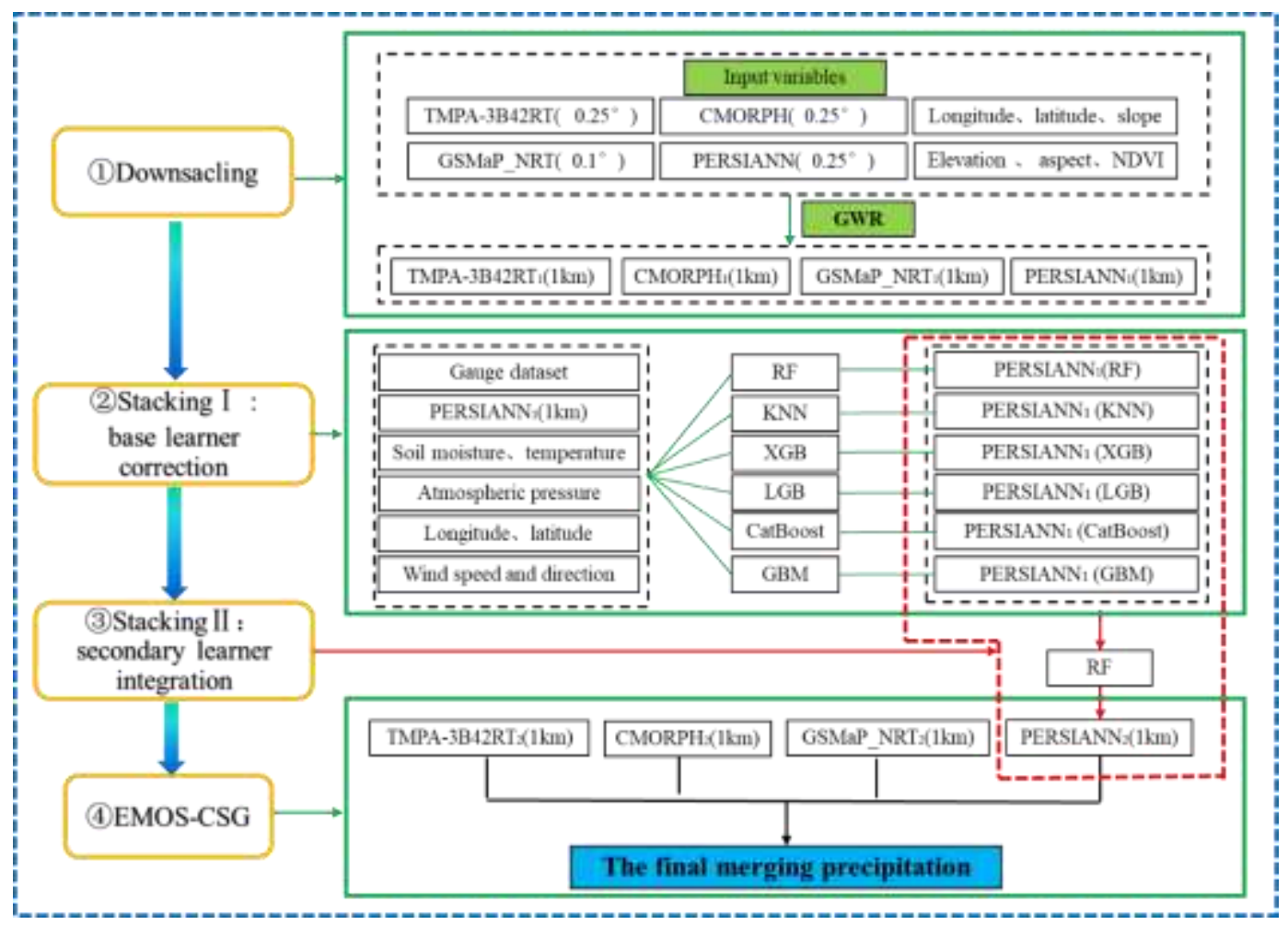




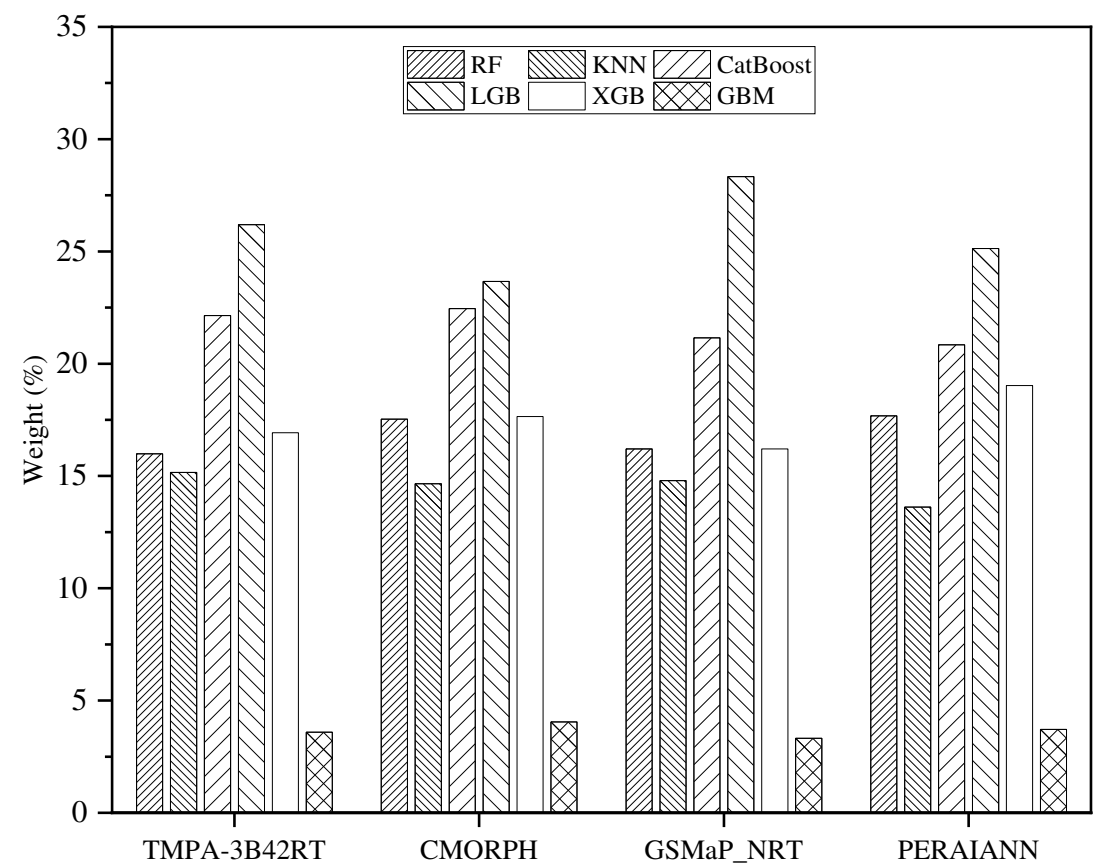

601

602 Fig. 3 Weights of the six machine learning algorithms applied to each product in the secondary learner

(a) TMPA-3B42RT

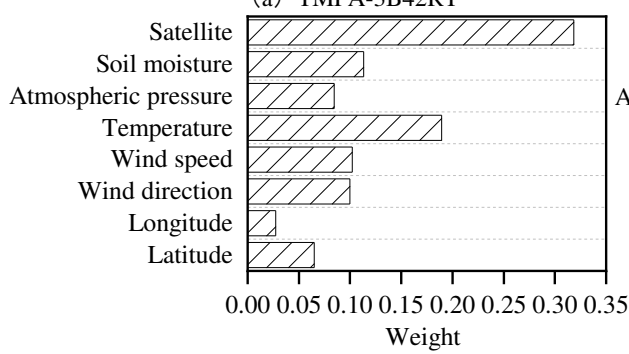

(b) CMORPH

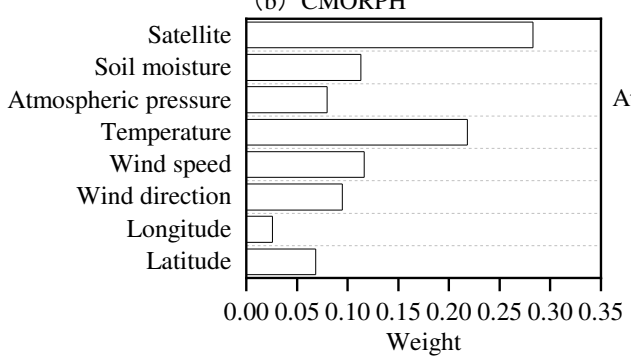

(c) GSMaP_NRT

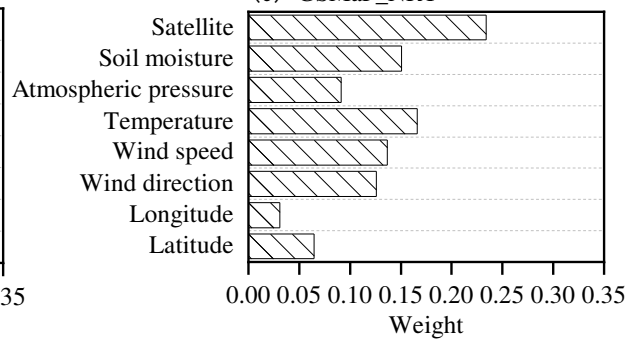

(d) PERSIANN

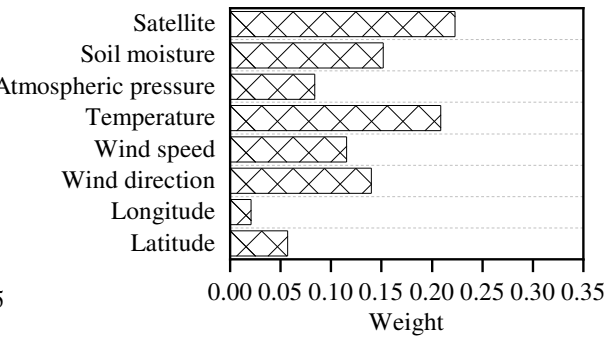

Fig. 4 Weights of each variable in the training dataset applied to the RF algorithm 

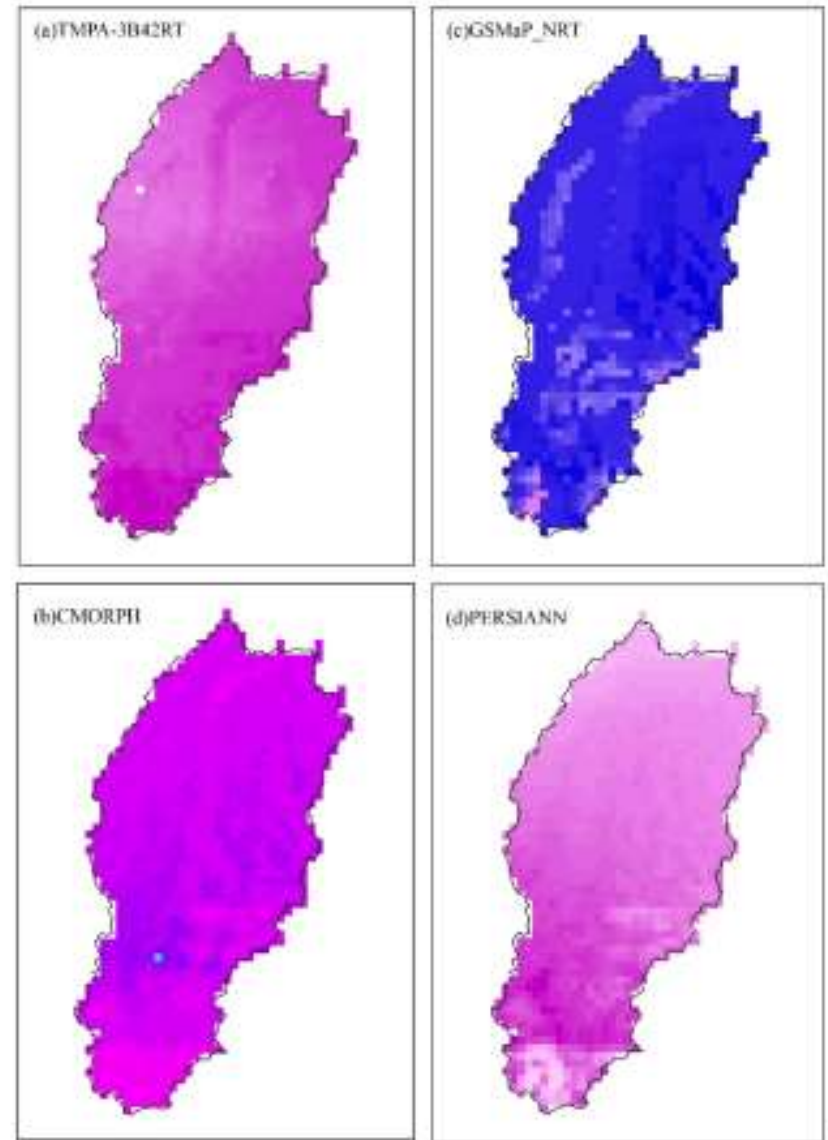

(ex)Mreing protuas

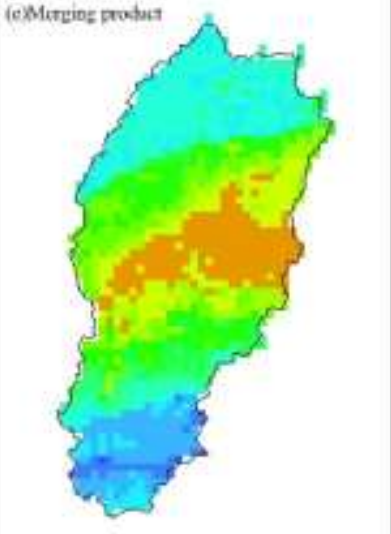

inGauge obsen atio

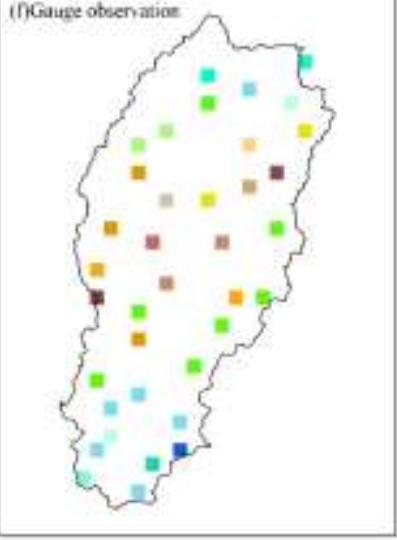

L.epgead

Fig. 5 Spatial distributions of precipitation obtained from the (a) downscaled TMPA-3B42RT product, (b) downscaled CMORPH product, (c) downscaled GSMaP_NRT product, (d) downscaled PERSIANN product, (e) merged product, and (f) gauge network on June 20, 

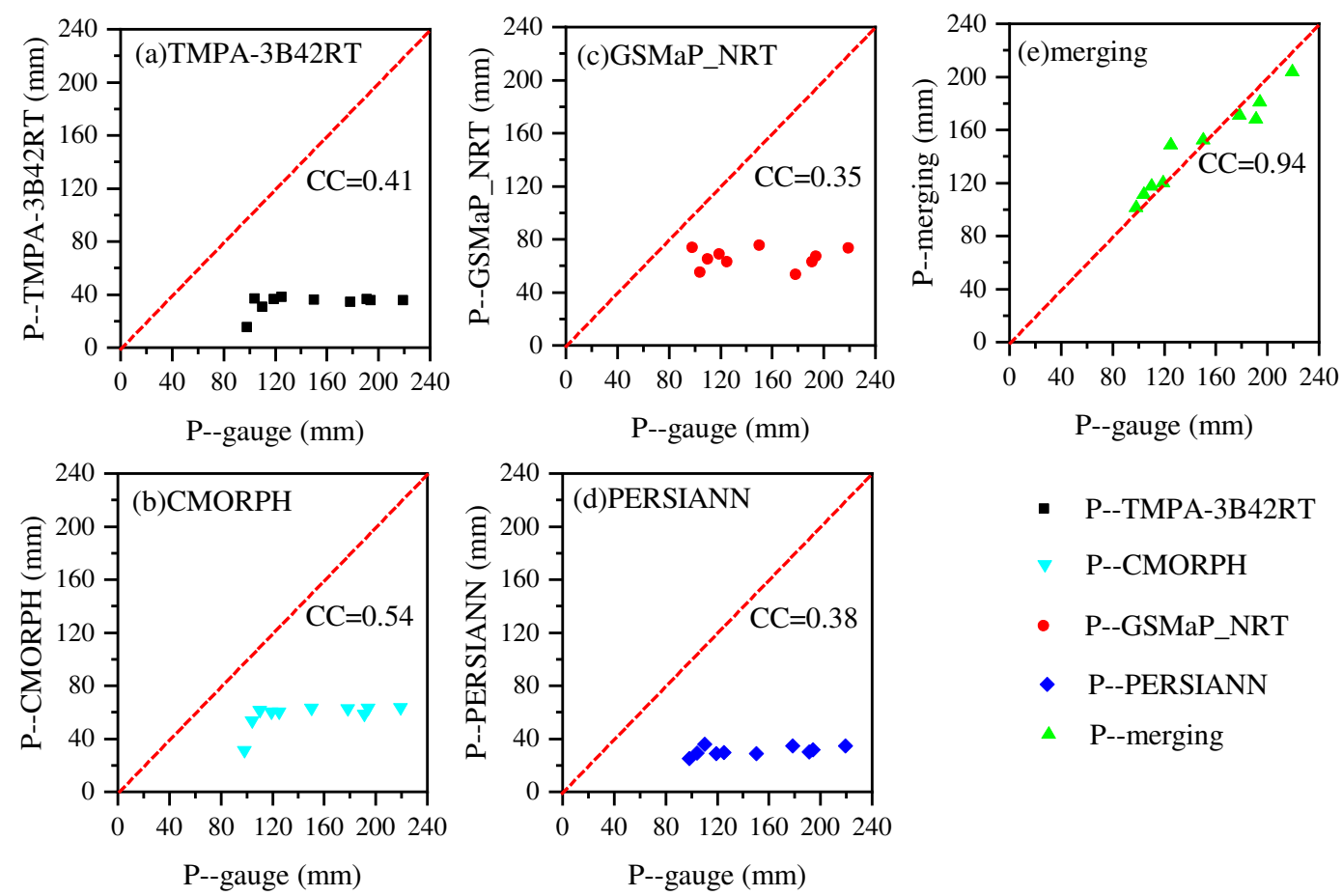

- P--TMPA-3B42RT

$\checkmark$ P--CMORPH

- P--GSMaP_NRT

- P--PERSIANN

$\triangle \quad$ P--merging

Fig. 6 Scatter plots of the gauge- and satellite-derived precipitation measured for (a) the downscaled TMPA-3B42RT product, (b) downscaled CMORPH product, (c) downscaled GSMaP_NRT product, (d) downscaled PERSIANN product, and (e) merged products on June 20, 2017
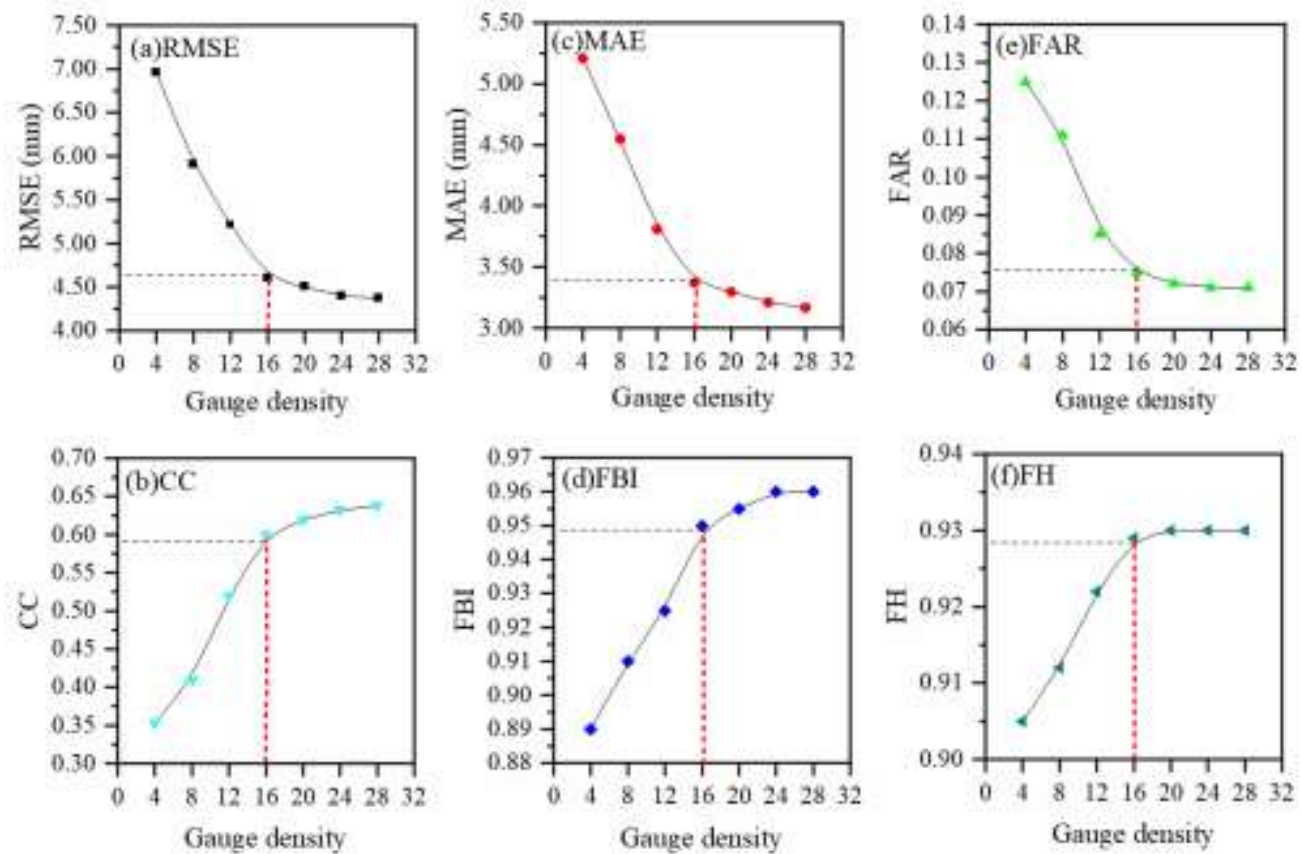

Fig. 7 Merging accuracy statistics obtained under different rainfall station densities 
Tab. 1 Spatiotemporal resolutions of remote sensing data and sources

\begin{tabular}{|c|c|c|}
\hline Product & $\begin{array}{c}\text { Spatiotemporal } \\
\text { resolution }\end{array}$ & Data source \\
\hline TMPA-3B42RT & $0.25^{\circ} / 3 \mathrm{~h}$ & https://gpm.nasa.gov/data/directory \\
\hline CMORPH & $0.25^{\circ} / 3 \mathrm{~h}$ & https://rda.ucar.edu/datasets/ds502.1/index.html\#!access \\
\hline GSMaP_NRT & $0.1 \% \mathrm{~h}$ & https://sharaku.eorc.jaxa.jp/GSMaP_CLM/index.htm \\
\hline PERSIANN & $0.25^{\circ} / 3 \mathrm{~h}$ & https://chrsdata.eng.uci.edu/ \\
\hline NDVI & $1 \mathrm{~km} / 1 \mathrm{~m}$ & https://ladsweb.modaps.eosdis.nasa.gov/search/ \\
\hline Soil moisture & $9 \mathrm{~km} / 3 \mathrm{~h}$ & https://nsidc.org/data/SPL4SMGP/versions/4 \\
\hline DEM & $90 \mathrm{~m}$ & http://www.gscloud.cn/ \\
\hline $\begin{array}{l}\text { Wind speed and direction, atmospheric } \\
\text { pressure, temperature }\end{array}$ & $0.1 \% \mathrm{~h}$ & $\begin{array}{c}\text { https://cds.climate.copernicus.eu/cdsapp\#!/dataset/reanalysis-er } \\
\text { a5-land?tab=overview }\end{array}$ \\
\hline
\end{tabular}

Tab. 2 Statistical metrics of original and downscaled satellite precipitation products

\begin{tabular}{cccccccc}
\hline Type & Product & RMSE $(\mathrm{mm})$ & CC & MAE $(\mathrm{mm})$ & FAR & FBI & FH \\
\hline \multirow{5}{*}{ Original } & TMPA-3B42RT & 12.20 & 0.23 & 10.55 & 0.19 & 0.70 & 0.81 \\
& CMORPH & 12.47 & 0.23 & 10.74 & 0.16 & 0.93 & 0.80 \\
& GSMaP_NRT & 12.59 & 0.21 & 11.00 & 0.17 & 0.95 & 0.79 \\
& PERSIANN & 12.55 & 0.13 & 11.01 & 0.19 & 0.88 & 0.80 \\
\hline \multirow{5}{*}{ Downscaled } & TMPA-3B42RT & 12.03 & 0.25 & 10.24 & 0.17 & 0.75 & 0.83 \\
& CMORPH & 12.39 & 0.25 & 10.52 & 0.15 & 0.95 & 0.82 \\
& GSMaP_NRT & 12.23 & 0.23 & 10.71 & 0.16 & 0.98 & 0.82 \\
& PERSIANN & 12.47 & 0.18 & 10.86 & 0.18 & 0.91 & 0.82 \\
\hline
\end{tabular}

632

633

634

Tab. 3 Evaluation statistics of the precipitation products used for merging

\begin{tabular}{cccccccc}
\hline Product & Algorithm & RMSE $(\mathrm{mm})$ & CC & MAE $(\mathrm{mm})$ & FAR & FBI & FH \\
\hline RF & 5.46 & 0.57 & 4.03 & 0.15 & 1.25 & 0.85 \\
KNN & 5.46 & 0.49 & 4.00 & 0.14 & 1.13 & 0.85 \\
TMPA-3B42RT & XGB & 5.47 & 0.54 & 4.00 & 0.16 & 1.39 & 0.84 \\
& LGB & 5.16 & 0.58 & 3.73 & 0.13 & 1.13 & 0.85 \\
& CatBoost & 5.18 & 0.51 & 3.95 & 0.17 & 1.28 & 0.83 \\
& GBM & 6.40 & 0.46 & 5.08 & 0.16 & 1.16 & 0.84 \\
& Stacking & 4.95 & 0.60 & 3.59 & 0.11 & 1.10 & 0.87 \\
\hline RF & 5.13 & 0.58 & 3.84 & 0.14 & 1.25 & 0.86 \\
& KNN & 5.49 & 0.50 & 4.01 & 0.13 & 1.12 & 0.87 \\
& XGB & 5.10 & 0.57 & 3.73 & 0.14 & 1.16 & 0.86 \\
& LGB & 5.00 & 0.59 & 3.64 & 0.12 & 1.12 & 0.87 \\
& CatBoost & 5.02 & 0.56 & 3.77 & 0.14 & 1.20 & 0.86 \\
& GBM & 6.29 & 0.46 & 4.90 & 0.16 & 1.22 & 0.83 \\
& Stacking & 4.80 & 0.60 & 3.46 & 0.11 & 1.11 & 0.89 \\
\hline GSMaP_NRT & RF & 5.35 & 0.56 & 3.96 & 0.14 & 1.21 & 0.86
\end{tabular}




\begin{tabular}{cccccccc} 
& KNN & 5.39 & 0.52 & 3.90 & 0.13 & 1.09 & 0.87 \\
XGB & 5.27 & 0.53 & 3.86 & 0.15 & 1.22 & 0.85 \\
LGB & 5.04 & 0.60 & 3.70 & 0.12 & 1.11 & 0.88 \\
CatBoost & 5.14 & 0.53 & 3.84 & 0.15 & 1.26 & 0.85 \\
GBM & 6.25 & 0.44 & 4.83 & 0.15 & 1.18 & 0.85 \\
& Stacking & 4.92 & 0.62 & 3.56 & 0.10 & 1.06 & 0.90 \\
\hline \multirow{3}{*}{ PERSIANN } & RF & 4.86 & 0.60 & 3.62 & 0.14 & 1.23 & 0.86 \\
& KNN & 5.12 & 0.56 & 3.74 & 0.13 & 1.12 & 0.87 \\
& XGB & 4.84 & 0.59 & 3.55 & 0.14 & 1.20 & 0.86 \\
& LGB & 4.78 & 0.60 & 3.55 & 0.13 & 1.14 & 0.87 \\
& CatBoost & 4.78 & 0.56 & 3.60 & 0.17 & 1.26 & 0.83 \\
Merged product & GBM & 6.20 & 0.44 & 4.90 & 0.17 & 1.21 & 0.83 \\
& Stacking & 4.53 & 0.62 & 3.31 & 0.10 & 1.09 & 0.90 \\
\hline
\end{tabular}

635

636

637

638

639

640 
